\title{
ANALYSIS OF DISTRIBUTED SPARSE ARRAY CONFIGURATIONS FOR GUIDED WAVE IMAGING APPLICATIONS
}

\author{
James S. Hall and Jennifer E. Michaels \\ School of Electrical and Computer Engineering, \\ Georgia Institute of Technology, Atlanta, Georgia 30332-0250
}

\begin{abstract}
Permanently attached, distributed sparse arrays of piezoelectric transducers have been proposed as a cost-effective solution for rapid interrogation of large, plate-like structures for structural health monitoring. Many proposed methods using these arrays rely on guided wave imaging techniques to interpret and graphically display information for damage detection and localization. Guided wave imaging algorithms, however, contain imaging artifacts that are due to reflections from boundaries and other structural features, even in a noise-free environment. The magnitude relationship between pixel values corresponding to actual damage and imaging artifacts is dependent on a number of variables, including imaging algorithm, number and location of transducers in the array, location and scattering behavior of damage, and structural geometry. This study proposes a metric to characterize the imaging performance of distributed sparse array systems and utilizes numerical simulations to analyze the impact of damage location, imaging algorithm, and physical array configuration on performance in the context of a single flaw in a rectangular aluminum plate.
\end{abstract}

Keywords: Ultrasonic, Lamb Waves, Nondestructive Evaluation, Structural Health Monitoring, Sparse Arrays, Array Geometry

PACS: 43.35.Zc, 43.60.Fg, 43.60.Lq, 43.60.Mn, 43.60.Pt.

\section{INTRODUCTION}

Guided waves are increasingly being used for interrogating large, plate-like structures for the purposes of nondestructive evaluation (NDE) and structural health monitoring (SHM) [1]. To obtain sufficient spatial information for detection and localization of structural defects, arrays of ultrasonic transducers are commonly employed to transmit and record guided waves. The proposed transducer arrays vary in terms of number of transducers, aperture size, and sensor pattern $[2,3]$.

Guided wave imaging algorithms are used to graphically present information obtained from an array of ultrasonic transducers. All images generated from guided wave imaging algorithms, including conventional delay-and-sum imaging [4,5] and MVDR imaging [6,7], include artifacts that result from energy that has reflected from both structural boundaries and internal scatterers. These imaging artifacts can severely degrade imaging performance and therefore the ability of an array to detect and localize damage. Several factors impact the sensitivity of a guided wave system to scattered and reflected 
energy: (1) the imaging algorithm, (2) the physical array configuration, such as aperture size, number of sensors, and sensor pattern, and (3) structural characteristics, such as structure geometry and damage scattering behavior.

After generating an image with a guided wave imaging technique, damage detection and localization can then be performed. Damage detection and localization present two distinctly different challenges that share substantial overlap. On one hand, damage localization identifies the most likely location of damage, typically by identifying the maximum pixel value in an image. The location of the maximum pixel value in an image, however, does not necessarily indicate that damage is present in the structure; even a damage-free image will have a maximum pixel value. Damage detection, on the other hand, identifies whether or not damage is present in a structure and is typically accomplished by establishing a threshold that, if exceeded, indicates the presence of damage. These two challenges are interrelated for guided wave images since each pixel is calculated independently. If a threshold for damage detection can be established on a pixel-specific basis, the threshold is by definition location dependent, and damage localization is thus achieved automatically.

The concept of establishing pixel-specific thresholds for damage detection, which was recently proposed by Flynn and Todd [8,9], represents a departure from previous guided wave imaging performance metrics that are focused on image quality. These metrics typically characterize the performance of an array by comparing the pixel value at one or more potential damage locations to the pixel values at all non-damage locations for a single image. The use of pixel-specific thresholds, however, foregoes this requirement and provides a more complete picture of an array's ability to perform simultaneous damage detection and localization.

Since there are so many variables associated with characterizing array performance, the scope of this paper is restricted to a single defect in a specific structure. A performance ratio is first proposed to quantify array performance. Simulated data are then used to demonstrate the impact of the imaging algorithm and physical array configuration on the proposed performance ratio.

\section{PIXEL-SPECIFIC PERFORMANCE RATIO}

As mentioned above, damage detection is focused on determining whether or not damage is present in a structure. By performing damage detection on a pixel-specific basis, damage localization is automatically achieved. To perform damage detection, a pixelspecific threshold, $T_{x y}$, must be established that satisfies:

$$
\max _{i j \neq x y} P_{x y}\left(D_{i j}\right)<T_{x y}<P_{x y}\left(D_{x y}\right)
$$

where $P_{a b}\left(D_{g h}\right)$ is the pixel value at location $(a, b)$ when damage is present at $(g, h)$. To maximize robustness to system noise, model errors, etc., the following performance ratio should be as large as possible for all pixel locations, $(x, y)$ :

$$
\frac{P_{x y}\left(D_{x y}\right)}{\max _{i j \neq x y} P_{x y}\left(D_{i j}\right)} \text {. }
$$

In words, the ratio expressed in Eq. (2) is the pixel value at $(x, y)$ when damage is present at $(x, y)$ compared to the maximum pixel value at $(x, y)$ when damage is present somewhere else in the structure, referred to as the maximum artifact value at $(x, y)$. If Eq. (2) $>1$ for all pixel locations, then pixel-specific thresholds can be established throughout the Hall, J. S. and Michaels, J. E., AIP Conference Proceedings 1335, 859-866 (2011) 
structure and any pixel value that exceeds the pixel-specific threshold must also correspond to the most-likely damage location. Therefore, damage detection and localization can be automated with a single comparison between an image and set of pixel-specific thresholds.

Equation (2) was chosen for the purposes of this paper for convenience since the recorded signals are assumed to have zero noise, which allows for deterministic analysis. From a statistical perspective, however, it should be noted that the ratio presented in Eq. (2) has a direct impact on the probability of damage detection, false-detect, and miss for location $(x, y)$. Rather than use the ratio from Eq. (2), a statistical model could be alternatively employed that incorporates the probability of damage occurring at any given pixel location, as well as the associated costs of false-detects and misses. This is the general approach taken by Flynn and Todd [8,9]; however, their work effectively focuses on the pixel value when damage is present and does not consider imaging artifacts.

As can be observed in Eq. (2), calculation of the pixel-specific performance ratio requires simulation of the structure and array configuration for every possible damage location. This can be extremely computationally demanding when attempting to identify an array configuration that optimizes the pixel-specific performance ratio. As such, only the most demanding of applications (such as high-performance aerospace or space-borne) are likely to merit an extensive search for optimal array geometries. The cases presented here, however, are intended to demonstrate to the reader those factors that most strongly influence array performance.

\section{FACTORS INFLUENCING ARRAY PERFORMANCE}

This section identifies the factors that most strongly influence the pixel-specific performance ratio for guided wave arrays. The factors can be grouped into three categories: (1) the imaging algorithm employed to analyze recorded signals, (2) the physical characteristics of the array, such as aperture size, number of transducers, and sensor pattern, and (3) the geometry of the structure and defect.

To demonstrate the relative impact of the first two factors, the pixel-specific performance ratio discussed in the previous section is calculated and displayed for two different imaging algorithms and several array configurations; consideration of the third factor is limited to qualitative comments. Simulations are performed in a noise-free environment for a $610 \mathrm{~mm} \times 610 \mathrm{~mm} \times 3 \mathrm{~mm} 6061$ aluminum plate and a point-like defect that radiates incident energy uniformly in all directions. Wave propagation was simulated with identical transducers, no dispersion, a group velocity of $5.5 \mathrm{~mm} / \mu \mathrm{s}$, and perfectly reflecting boundaries. The guided wave was simulated as a three-cycle Hanning windowed sinusoid at $250 \mathrm{kHz}$, which corresponds to a $66 \mathrm{~mm}$ long wave-packet. Signals were simulated for each unique pair of transducers. For example, six signals were simulated for an array of four transducers, and 15 signals for an array of six transducers.

Performance ratios were obtained by simulating the wave propagation and calculating images for a single defect located on a $20 \mathrm{~mm}^{2}$ grid. Since defect locations are restricted to grid locations, $P_{x y}\left(D_{x y}\right)$ is independent of the defect grid resolution; however, $\max _{i j \neq x y} P_{x y}\left(D_{i j}\right)$ is not. Thus, as grid spacing is reduced, performance ratios will either remain at the same levels or decrease. Therefore defect grid resolution must be sufficiently small to ensure that the resulting performance ratios are not overly optimistic.

To aid in analysis, pixel values adjacent to transducers or defect locations were ignored when computing the denominator of Eq. (2). The large pixel values associated with these locations prevent useful data from being extracted from the performance ratio maps. All pixel-specific performance ratios are shown on a dB scale. 


\section{Imaging Algorithm}

A guided wave imaging algorithm is used to graphically present the information recorded from a guided wave transducer array. This paper considers two elliptical imaging algorithms that calculate each pixel value independently, specifically conventional delayand-sum imaging [4,5] and MVDR imaging [6,7]. These imaging algorithms are briefly summarized here to orient the reader. More detailed explanations and discussions can be found in the referenced literature.

To isolate energy scattered from a defect, known damage-free baseline signals are subtracted from each recorded signal prior to analyzing the data. For this paper, it is assumed that perfect baseline subtraction is achieved and the signals used for imaging consist of only the scattered energy.

The equation used to calculate each pixel is:

$$
P_{x y}=\overrightarrow{\mathbf{w}}_{x y}^{\mathrm{H}} \mathbf{R}_{x y} \overrightarrow{\mathbf{w}}_{x y}
$$

where $P_{x y}$ is the pixel value for location $(x, y), \overrightarrow{\mathbf{w}}_{x y}$ is a pixel-specific vector of weights, the superscript " $\mathrm{H}$ " indicates a Hermetian transpose operation, and $\mathbf{R}_{x y}$ is a correlation matrix. The correlation matrix, $\mathbf{R}_{x y}$, is computed as:

$$
\mathbf{R}_{x y}=\int_{t_{1}}^{t_{2}} \overrightarrow{\mathbf{r}}_{x y}(t) \overrightarrow{\mathbf{r}}_{x y}^{\mathrm{H}}(t) d t,
$$

where $\overrightarrow{\mathbf{r}}_{x y}(t)$ is a vector of recorded signals that have been back-propagated according to the propagation distance. More specifically,

$$
\overrightarrow{\mathbf{r}}_{x y}(t)=\left[s_{1}\left(t+\frac{d_{1 x y}}{c_{\mathrm{g}}}\right) \quad \cdots \quad s_{M}\left(t+\frac{d_{M x y}}{c_{\mathrm{g}}}\right)\right]^{\mathrm{T}},
$$

where $c_{\mathrm{g}}$ is the group velocity $\left(5.5 \mathrm{~mm} / \mu\right.$ s for this paper), $s_{i}(t)$ is the $i$ th recorded signal, $M$ is the number of recorded signals, and $d_{i x y}$ is the total distance from the transmitter to location $(x, y)$ to the receiver for the $i$ th recorded signal. Hall and Michaels [6] demonstrated that for high SNR environments, such as that considered here, the integration interval in Eq. (4) can be reduced to a single time-sample corresponding to the peak of the transmitted signal. Therefore, all simulations are performed using a single time-sample.

The weight vectors, $\overrightarrow{\mathbf{w}}_{x y}$, from Eq. (3) represent the fundamental difference between the two imaging algorithms considered here. For conventional delay-and-sum imaging, the weight vector is a unit-norm vector selected to maximize the pixel value when damage is present. The delay-and-sum weight vector is:

$$
\overrightarrow{\mathbf{W}}_{x y} \sim\left[\begin{array}{ccc}
1 / \sqrt{d_{1 x y}^{\times}} & \cdots & 1 / \sqrt{d_{M x y}^{\times}}
\end{array}\right]^{\mathrm{T}},
$$

where $d_{i x y}^{\times}$is the product of distances from transmitter to pixel location $(x, y)$, and pixel location $(x, y)$ to receiver for the $i$ th transducer pair and is used to account for the expected decay in signal strength due to geometric spreading. Figure 1 shows pixel-specific performance ratios for a rectangular array pattern with four, six, and eight sensors using conventional delay-and-sum imaging. 


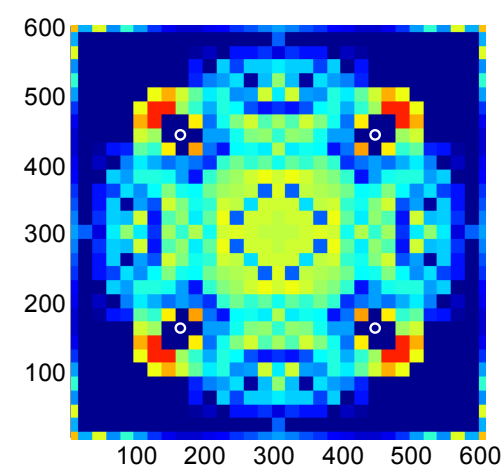

(a)

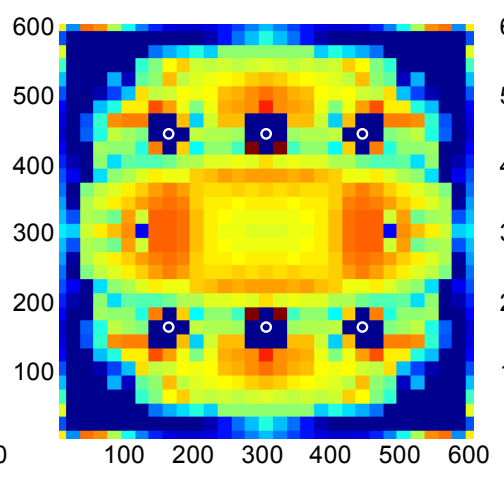

(b)

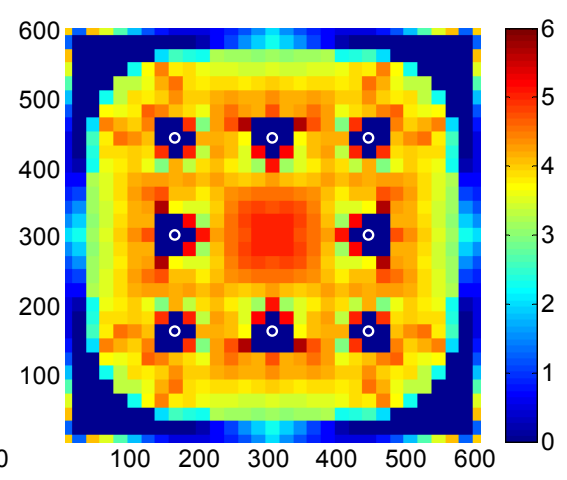

(c)

FIGURE 1. (Color online) Performance ratio maps generated with conventional delay-and-sum imaging and a four, six, and eight sensor rectangular pattern. Sensors are indicated with white 'o' symbols, and the color scale is in $\mathrm{dB}$ with the lower limit fixed at $0 \mathrm{~dB}$.

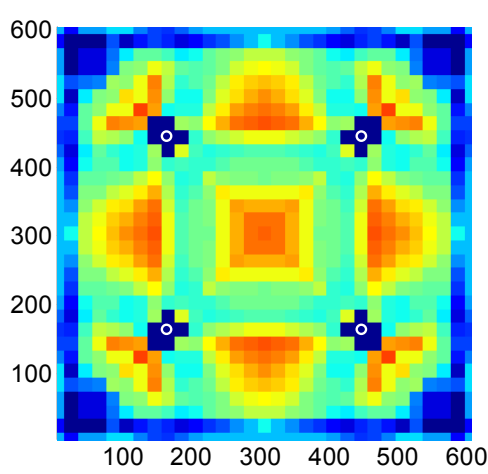

(a)

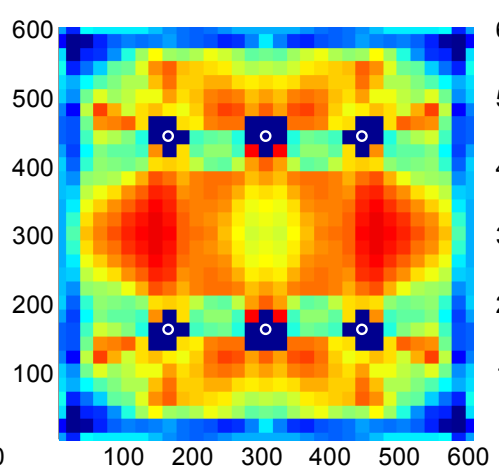

(b)

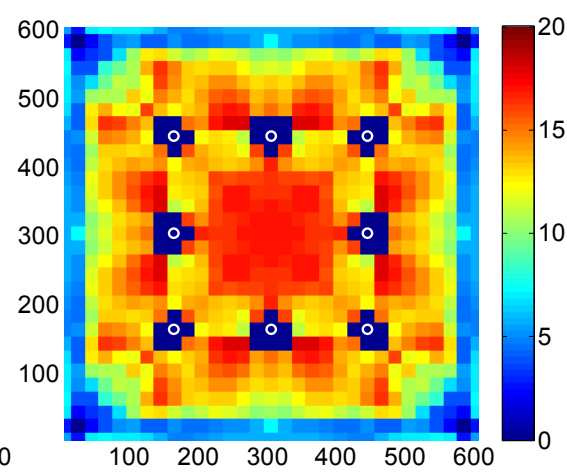

(c)

FIGURE 2. (Color online) Performance ratio maps generated with MVDR imaging and a four, six, and eight-sensor rectangular pattern.

Alternatively, the weight vector used for MVDR imaging is selected to minimize all pixel values subject to a constraint that preserves the pixel value at damage locations. The MVDR weight vector is calculated as:

$$
\overrightarrow{\mathbf{w}}_{x y}=\frac{\mathbf{R}_{x y}^{-1} \mathbf{e}_{x y}}{\overrightarrow{\mathbf{e}}_{x y} \mathbf{R}_{x y}^{-1} \overrightarrow{\mathbf{e}}_{x y}},
$$

where the steering vector, $\overrightarrow{\mathbf{e}}_{x y}$, is identical to $\overrightarrow{\mathbf{w}}_{x y}$ from Eq. (6). Since the correlation matrix, $\boldsymbol{R}_{x y}$, is computed as in Eq. (5) with a single time sample, the matrix inversion is illposed. Diagonal loading is used to regularize the matrix inversion of Eq. (7), as in Hall and Michaels [6], with 0.1 times the squared norm of $\overrightarrow{\mathbf{r}}_{x y}(\tau)$ where $\tau$ is the time corresponding to the peak of the transmitted signal. Figure 2 shows pixel-specific performance ratios for the same four, six, and eight sensor arrays as in Figure 1 using MVDR imaging.

The imaging algorithm strikes a balance between sensitivity to the direct arrival of scattered energy and robustness to reflections of scattered energy (and errors in a priori information, which is beyond the scope of this text). Comparing Figure 2 to Figure 1, MVDR imaging clearly offers significant improvements over conventional delay-and-sum imaging for these specific array configurations. This improvement is expected, since the MVDR weight vectors are selected specifically to reduce the pixel value when the backpropagated signals do not agree with the steering vector, $\overrightarrow{\mathbf{e}}_{x y}$. What may not be expected is the degree of improvement. The maximum performance ratio is approximately $6 \mathrm{~dB}$ in Figure 1, while Figure 2 exhibits performance ratios in excess of $20 \mathrm{~dB}$. Similarly, Hall, J. S. and Michaels, J. E., AIP Conference Proceedings 1335, 859-866 (2011) Review of Progress in Quantitative Nondestructive Evaluation, Volume 30 


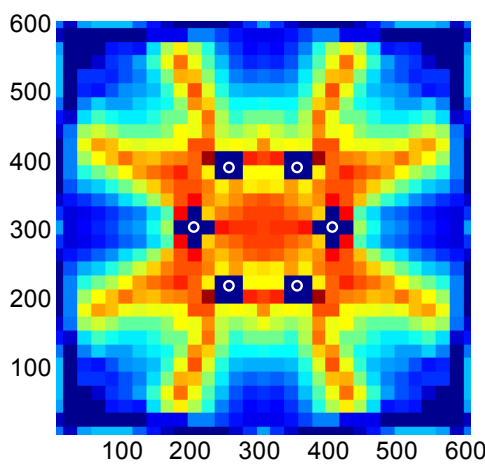

(a)

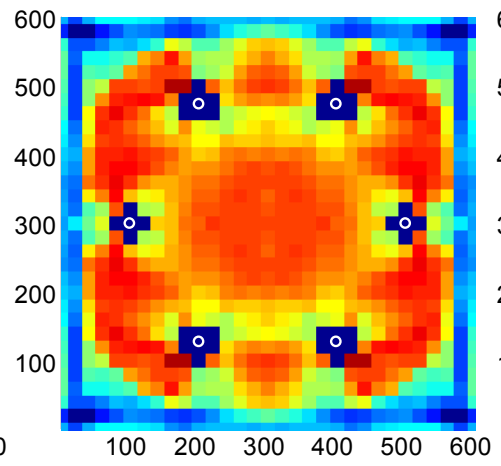

(b)

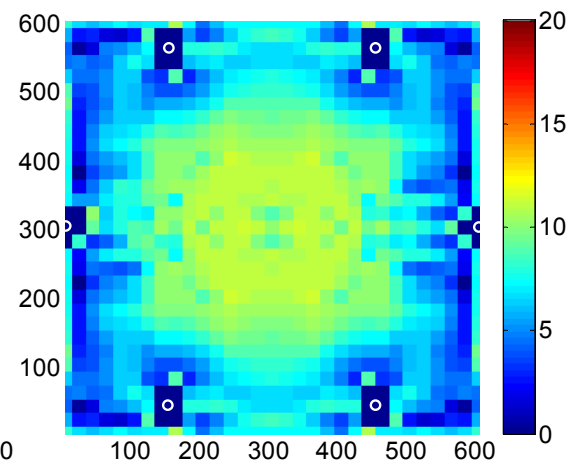

(c)

FIGURE 3. (Color online) Performance ratio maps with MVDR imaging using a 6-sensor circular array with $100 \mathrm{~mm}, 200 \mathrm{~mm}$, and $300 \mathrm{~mm}$ radius aperture size.

although all six plots in Figures 1 and 2 exhibit some performance degradation near the edges of the structure, the degradation is much more pronounced for conventional delayand-sum imaging. As such, the pixel-specific performance ratio is less than $1(<0 \mathrm{~dB})$ for many parts of the structure, which means that some pixel locations will exhibit a lower pixel value when damage is present at that pixel location than when damage is present elsewhere in the structure. Alternatively, for the array geometries considered in Figures 1 and 2, the MVDR imaging algorithm consistently provides pixel-specific performance ratios much greater than 1 for the vast majority of the structure.

\section{$\underline{\text { Physical Array Configuration }}$}

The physical array configuration also has a significant impact on performance. The array configuration is characterized by the aperture size, number of sensors, sensor pattern, and orientation of the array relative to the structure. In effect, the array configuration determines the spatial information that is obtained from the structure. An exhaustive study of all possible configurations is clearly impossible, so a small subset of examples have been chosen to demonstrate typical performance impacts from each of these factors.

Aside from the imaging algorithm, the array aperture size appears to have the most significant impact. Figure 3 demonstrates pixel-specific performance ratios using MVDR imaging for six-sensor circular arrays with radii of (a) $100 \mathrm{~mm}$, (b) $200 \mathrm{~mm}$, and (c) $300 \mathrm{~mm}$ (in addition, a six-sensor circular array with a radius of $12 \mathrm{~mm}$ is shown in Figure 5(c)). The performance ratio maps are clearly affected by aperture size. Although edge reflections of the scattered energy are present in all simulations, the $100 \mathrm{~mm}$ and $300 \mathrm{~mm}$ radii arrays appear to be more susceptible to these reflections than the $200 \mathrm{~mm}$ circular array. Figure 3 suggests that for a given application, sensor pattern, and interrogation structure, some optimal aperture size exists.

As expected, the number of sensors also plays a significant role in array performance. Figures 1 and 2 demonstrate the improvements associated with an increase in the number of sensors. As discussed in the previous section, unique transducer pairs are used for imaging. As such, the number of signals used for imaging is $N(N-1) / 2$, where $N$ is the number of sensors. Therefore doubling the number of sensors from four to eight sensors increases the number of recorded signals from 6 to 28 . One observation that can be made from Figures 1 and 2 is that the number of sensors appears to improve the performance map spatially, but does not seem to improve the maximum performance ratio for the array configuration. 


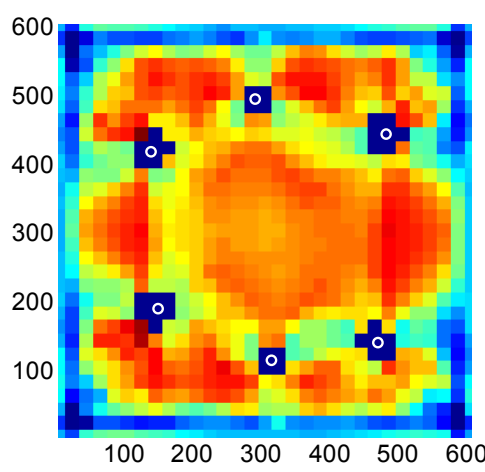

(a)

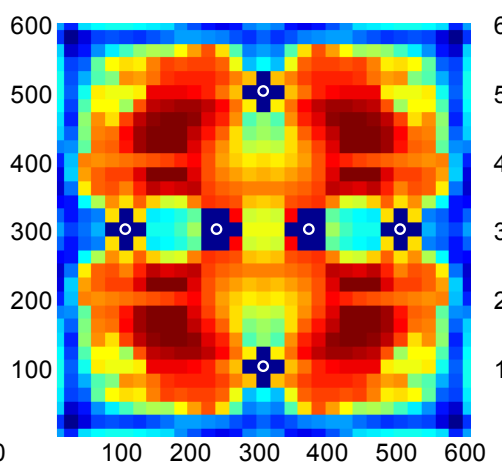

(b)

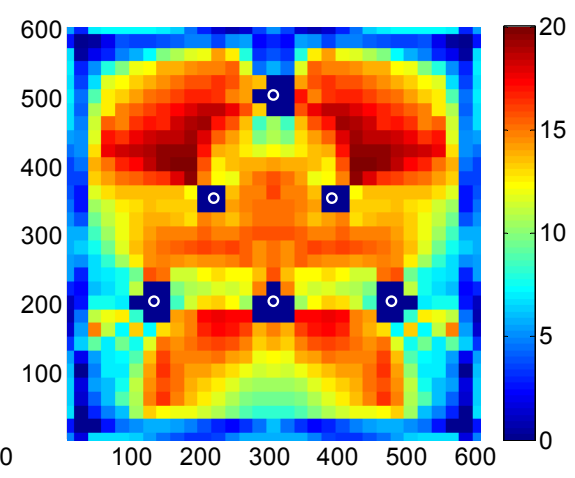

(c)

FIGURE 4. (Color online) Performance ratio maps with MVDR imaging using a 6-sensor array arranged in (a) a perturbed circular pattern, (b) a cross pattern, and (c) a triangular pattern.

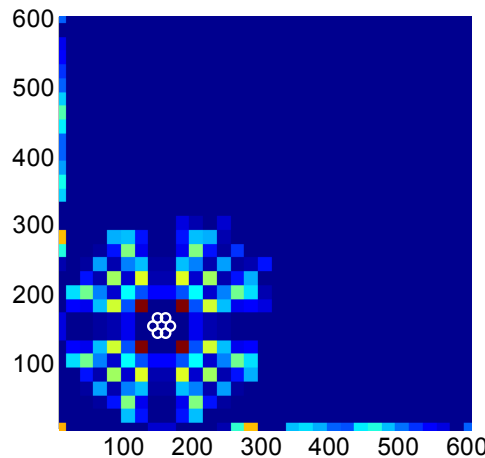

(a)

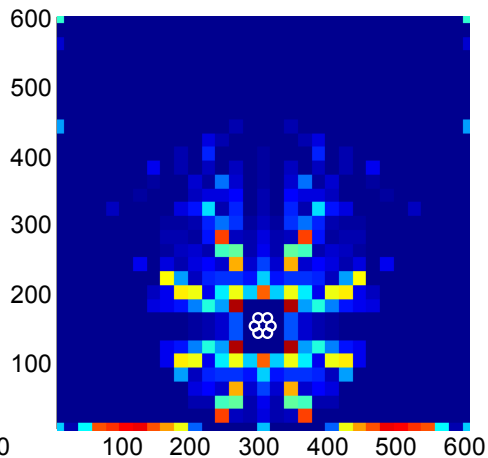

(b)

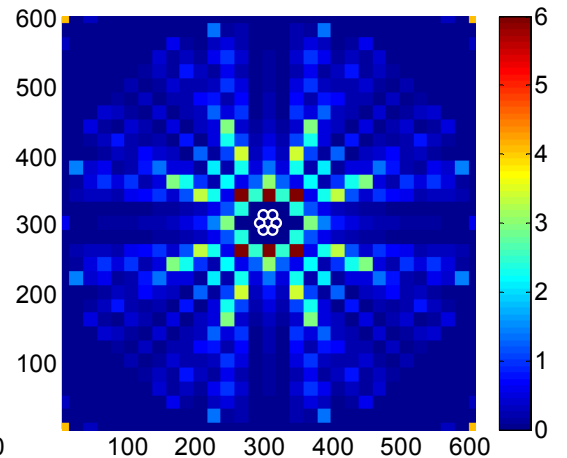

(c)

FIGURE 5. (Color online) Performance ratio maps with MVDR imaging using a compact 6-sensor array at three different locations on the interrogation structure.

Figures 2(b), 3(b), and 4(a-c) demonstrate five different sensor patterns for a six sensor array with approximately the same aperture sizes. Sensor patterns that exhibit spatial periodicity that resembles that of the interrogation structure can be expected to exhibit spatially periodic performance ratios. This can be observed throughout Figure 2, where the spatial periodicity of the performance ratio is strongest. Similar to the number of sensors, the sensor pattern appears to affect the spatial distribution of performance rather than the peak performance.

Finally, the location and orientation of an array with respect to the interrogation structure must be considered. Figure 5 shows the performance ratio for a six-sensor circular array with a $12 \mathrm{~mm}$ radius at three different locations on the structure. For this particular structure, the imaging performance is clearly improved by centering the array in the structure; however, this observation may not hold for all structures. Figure 5 indicates that optimal placement of a small array does little to improve array performance compared to the benefits of increasing the array aperture.

\section{$\underline{\text { Structure and Defect Characteristics }}$}

In the previous section, the aperture size, number of sensors, sensor pattern, and array location were all shown to affect array performance. It is important to note, however, that these characteristics are all dependent on the interrogation structure. A structurally centered circular array of a specific radius may be optimal for one structure, yet perform miserably for another. This is because the performance ratio proposed in 
Eq. (2) is largely driven by the imaging artifacts, which are produced by scattered energy reflected from geometric features associated with the interrogation structure.

In addition to the interrogation structure, the scattering behavior of defects is also expected to play a noticeable role in array performance. Defects that exhibit highly directional scattering patterns will not distribute energy in the same manner as point-like scatterers and, as such, the pixel values and performance ratios will vary with scattering characteristics. This concept is explored in more detail by Fromme [10].

\section{CONCLUSIONS}

Imaging artifacts are an inherent part of guided wave imaging algorithms. Depending on the algorithm, physical array configuration, and interrogation structure, imaging artifacts at some non-damage locations may be stronger than the pixel value at actual damage locations. Since this can prevent damage detection and localization, such artifacts must be considered when evaluating sparse array system performance.

A pixel-specific performance ratio has been defined that is the ratio of the pixel value when damage is present at that location to the maximum artifact value at that pixel location when damage is present elsewhere in the structure. Although the use of a pixelspecific ratio for array performance characterization can be computationally demanding, requiring the simulation of all possible damage types and locations, it provides a clear indication of how robust the array configuration is to system noise and modeling errors and whether or not simultaneous damage detection and localization can be performed.

Guided wave imaging algorithms and array aperture size were shown to have the most significant impact on array performance for the cases considered. MVDR imaging consistently provided performance ratios that were almost an order of magnitude better than conventional delay-and-sum imaging. The number of sensors and the sensor pattern were also shown to impact performance ratios, although not to the same degree as imaging algorithm and aperture size. Finally, although not demonstrated in this paper, the interrogation structure and defect scattering characteristics are also expected to impact array performance since these factors strongly influence imaging artifacts.

Future work remains in incorporating the impact of imaging artifacts into statistical models for determining optimal or near-optimal array configurations as well as investigating more efficient methods for comparing expected array performance.

\section{ACKNOWLEDGEMENTS}

The authors would like to thank NASA's Graduate Student Research Program (GSRP) for their support through Grant No. NNX08AY93H, and the Air Force Office of Scientific Research (AFOSR) for their support through Grant No. FA9550-08-1-0241.

\section{REFERENCES}

1. V. Giurgiutiu, Structural Health Monitoring with Piezoelectric Wafer Active Sensors. (Elsevier Academic Press, 2008).

2. A. J. Croxford, P. D. Wilcox, and B. W. Drinkwater, "Guided wave SHM with a distributed sensor network," Proc. SPIE, 6935 (69350E) pp. 1-9 (2008).

3. A. J. Croxford, P. D. Wilcox, and B. W. Drinkwater, "Quantification of sensor geometry performance for guided wave SHM," Proc. SPIE, 7295 (72951H) pp. 1-10 (2009).

4. C. H. Wang, J. T. Rose, and F.-K. Chang, "A synthetic time-reversal imaging method for structural health monitoring," Smart Mater. Struct., 13 pp. 415-423 (2004).

5. J. E. Michaels and T. E. Michaels, "Guided wave signal processing and image fusion for in situ damage localization in plates," Wave Motion, 44 (482-492) (2007). 
6. J. S. Hall and J. E. Michaels, "Minimum variance ultrasonic imaging applied to an in situ sparse guided wave array," IEEE Trans. Ultrason., Ferroelectr., Freq. Control, (in press) (2010).

7. J. E. Michaels, J. S. Hall, G. Hickman, and J. Krolik, "Sparse array imaging of change-detected signals by minimum variance processing," in Review of Progress in QNDE, 28, pp. 642-649 (2009).

8. E. B. Flynn and M. D. Todd, "A Bayesian approach to optimal sensor placement for structural health monitoring with application to active sensing," Mech. Syst. Signal Pr., 24 pp. 891-903 (2010).

9. E. B. Flynn and M. D. Todd, "Optimal placement of piezoelectric actuators and sensors for detecting damage in plate structures," J. Intel. Mat. Syst. Str., 21 pp. 265-174 (2010).

10. P. Fromme, "SHM of large structures using guided waves for crack detection," in Review of Progress in QNDE, 30, (2011). 